\title{
Estimation of Body Size by Combining Genetic Algorithm with Human Body Model
}

\author{
Nurbiya Yadikar ${ }^{1}$, Shujing Zhang ${ }^{1}$, Hornisa Mamat ${ }^{1}$, Mutallip Mamut ${ }^{2}$ and Kurban Ubul ${ }^{1, *}$ \\ ${ }^{1}$ School of Information Science and Engineering, Xinjiang University, Urumqi, 830046 China \\ ${ }^{2}$ The Library of Xinjiang University, Urumqi, 830046 China \\ *Corresponding author
}

\begin{abstract}
Estimation of body size using related dataset is one of the challenging tasks in modeling and simulation area. It has a wide range of utilization in many aspects in society including body modeling, designing clothes etc. It is necessary to extract feature data in body size measurement. Except ordinary data such as the body height, weight and chest size, the body surface area and volume were made up B-Spline surface shape model by linear combined with these data in this paper. Several control points were selected after using Genetic Algorithm (GA) to select best points in theses dataset, and mathematical model of estimating human body size was created. Experimental results indicated that this model has advantages of high efficiency and low error rates in estimating human body size.
\end{abstract}

Keywords-genetic algorithm: GA; surface area; the control points of b-spline surface

\section{INTRODUCTION}

The research of human body shape is one of the important research fields of ergonomics [1], which contains all kinds of information. In the field of clothing, body shape plays an important role as non-verbal information. Especially, from the clothing silhouette curve point of view, women's underwear, swimwear and other personal clothing needs more in-depth study of the human body shape. It is also important to grasp your body shape when designing clothes. In order to use the data of the body shape flexibly in the design of clothes, the 3D body model is made by 3D data of the body shape. Generally, in the process of designing clothes, anthropometric method [2] is a necessary method for extracting data of body shape.

In order to the human body model, it has been a lot of research results. Jones [3] first used laser 3D scanner to measure body shape. Li Jian et al. [4] measured and extracted human characteristics using a Harris corner detection method. Li Fu-xing et al. [5] proposed a new method of human body size correction and reconstruction. The literature [6] was the use of semantic and geometric characteristics of human body model structure. There was also a human body model created by extent of body [7] and convolutional neural networks [8].In this paper, B-Spline surface control points and Genetic Algorithms are used to derive size formulas for creating human models.

In the paper, the size was calculated by the linear combination of B-spline surface control points [9], which constitutes the body shape model. Genetic Algorithm (GA) [10] is a kind of randomized search method which is based on the evolution of biology. And it is the best method for the model of biological evolution and genetics. According to this algorithm, the arrangement of the optimal control points used in the calculation formula is analyzed.

Five difference [11] that separate genetic algorithms from conventional optimization techniques are concluded: 1) Operate the coding of the parameters; 2) The search starts from many points, rather than starting for a point, and it is effectively prevent the search converges to the local optimal solution; 3) Calculate the fitness value by the objective function, less dependency on the problem; 4) The optimization rule is determined by the probability; 5) Do efficient heuristic search in the solution space, rather than the blind exhaustive or completely random search. Based on the advantages of Genetic Algorithm, it has been applied in economy, management and other fields to varying degrees.

In the paper, the derivation method of control points coordinates is used to obtain the height, waistline, weight, and frequently changing data (body surface area, volume) and derives the calculation formula. Finally, the obtained formula is verified.

\section{THE SIZE OF THE EXPERIMENTAL OBJECT}

In the anthropometric data, in addition to the height, waistline, weight, other items are difficult to measure directly. The human body surface area and volume can be calculated by the data of human body model. In this way, the size valve of the above five items are used as the calculation object. Moreover, because the 3D model is only the main part body, the surface area and volume of the three-dimensional main part body model is derived.

The subjects are 85 adult women. According to the data of 60 human body models, the size calculation formula is analyzed. The size formula is derived for the remaining 25 persons, and the practicability of the size formula is analyzed.

In the analysis, if the error between "the measured value of the objects' body size" and "the calculated (regression) value obtained from the size value calculation formula" is small, the calculation formula is reliable. On the other hand, after the size value of calculation formula is obtained from the remaining 25 people, if the error is smaller, it can consider that the formula can be used for the general population.

\section{Approximation of SURFAce AREA AND Volume}

The surface area is a measure of the surface size. For example, the sphere surface area is a measure of the sphere 
surface size. It is also a function of the sphere radius $\mathrm{R}$. Because the sphere is an undevelopable surface, surface area is still derived by the method which is "segmentation, the sum of approximations, and converted from the sum of the approximations to the sum of the exact values". When the surface area of the body is obtained, the surface area is subdivided, and the model surface area is approximated by the sum of the planar areas obtained before the model surface is assumed to be plane. The control point is the parameter that describes the surface shape model in [9]. It can be used to calculate the size of a surface by a handful of control points. The linear combination formulas of B-Spline Surfaces control point is:

$$
P(u, v)=\sum_{i=1}^{m+3} \sum_{j=1}^{n} N_{i 3}(u) N_{j 3}(v) \boldsymbol{V}_{i j}
$$

where, $p(u, v)$ is the $3 \mathrm{D}$ coordinate value corresponding to the surface coordinates (u, v) on the model. $N$ is B-spline base coefficient of $i$ and $j . V_{i, j}$ is the coefficient that determines the shape of the B-spline surface and it is the control point on the surface. The body shape model is expressed by control point. When $m=30$, when $n=25$, B-spline surface shape model can approximate the human body model.

In the analysis model of the surface similar to Fig.1, it is surrounded by three points in the triangle. If the distance between the center point of the region and the plane formed by the three points is smaller than the threshold value, the surface of the model is regarded as a plane, and the area of the plane is the area of the two triangles. On the contrary, if the model surface cannot be regarded as a plane, then the plane 4 is divided equally to subdivide the model surface, and the surface area of the model is calculated by the sum of the obtained plane areas.

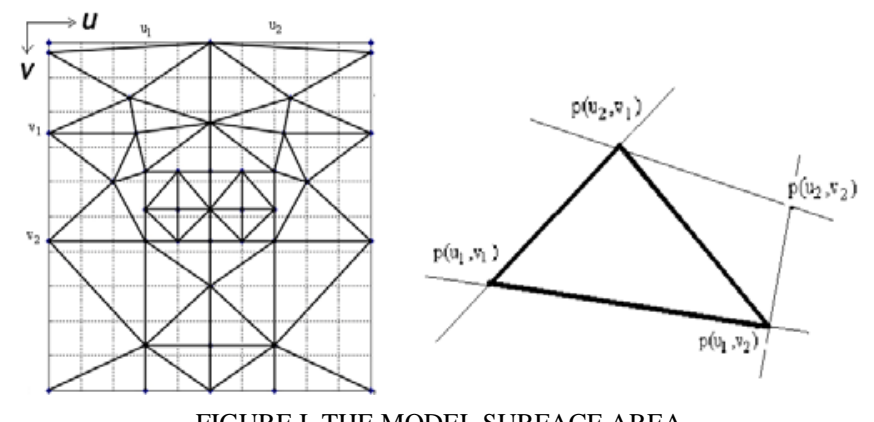

FIGURE I. THE MODEL SURFACE AREA

Volume refers to the size of space occupied by a substance or an object, which occupies a specific volume. For example, if a group of equidistant plane cut the sphere, a lot of "small rounds" are gotten by the wee distance, and the sum of "small rounds" volume is the sphere volume. The "Small round" is approximates cylindrical shape, so its volume is similar to the sum of the corresponding cylinder volume. Therefore, the sphere volume can be calculated by the method of "divided summed-translated into the sum of exact”.

As shown in Figure 2, the B-Spline Surface of the human body is divided into five facets, and the sum of the volume of the obtained pentahedron is similar to the model volume. The $u$ $v$ plane is respectively divided into $\mathrm{N}$ equal division in the $\mathrm{u}$ direction and the $\mathrm{v}$ direction.

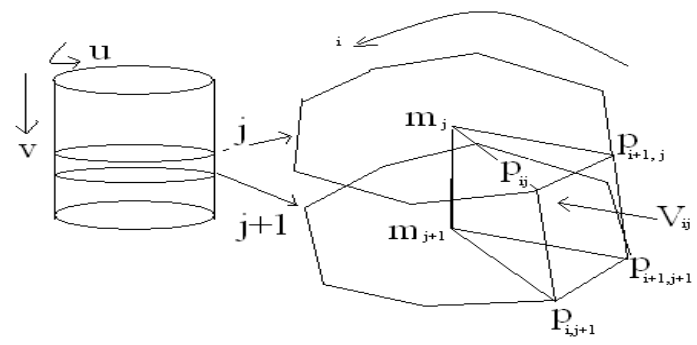

FIGURE II. APPROXIMATE VOLUME OF THE MODEL

If the number $\mathrm{N}$ of the division plane is enlarged, the volume of the model can be obtained with a high degree of accuracy.

\section{The Formula FOR CALCUlating the SizE AND GENETIC ALGORITHM}

\section{A. The formula of calculating the size value by GA}

In the genetic algorithm [10], the design variables are represented as genes on a chromosome. The chromosome represents the problem solution. In this algorithm, chromosome is a binary string representation. Before the implementation of genetic algorithm, a set of chromosomes are given, and these represent the hypothetical solution. Through environmental selection and the genetic operators, recombination and variation, chromosomes with better fitness are found. The evolution of such a generation, a chromosome is gotten which is the fittest environment. The chromosome is the optimal solution. The survival of the fittest guarantees that best chromosomes with better fitness will survive in the future populations. The chromosomes of the selected genes are used as biological individuals and play their roles.

In this paper, the performance characteristic is "linear formula based on chromosome (control point coordinates V)", which is used for "the size calculation ". Now,

$$
D=a_{0}+\sum_{i=1}^{n} a_{i} v_{i}
$$

When the measured size of the measured object is $\mathrm{S}^{\prime}, a_{i}(i$ $=0,1, \ldots \ldots n)$ is set in order to minimize in order to minimize the square sum of the error of the $\mathrm{N}$ subjects.

$$
\sum_{\text {Item }}\left\{D^{\prime}-D\right\}^{2}
$$

The minimum square sum is called the least square error. At this time, Q, which is the average error of each subject, is:

$$
Q=\sqrt{1 / N \sum_{N}\left\{D^{\prime}-D^{2}\right\}^{2}}
$$


When $a_{i}$ is sought for each generation, the value of $\mathrm{Q}$ and the correlation coefficient $\mathrm{R}$ of $\left\{D^{\prime}\right\}$ and $\{D\}$ can be used in the analysis of individual.

\section{B. Genetic Algorithm and Improved Genetic Algorithm}

Genetic algorithm [10] is the search algorithm used to solve the optimization problem in Computational Mathematics, and the algorithm uses binary code to represent the population, and the fitness is the evolutionary condition. In order to find the optimal solution, GA selects the optimal individual evolution to the next generation. It can easily solve the resource allocation problem with certain constraints, as shown in Figure 3.

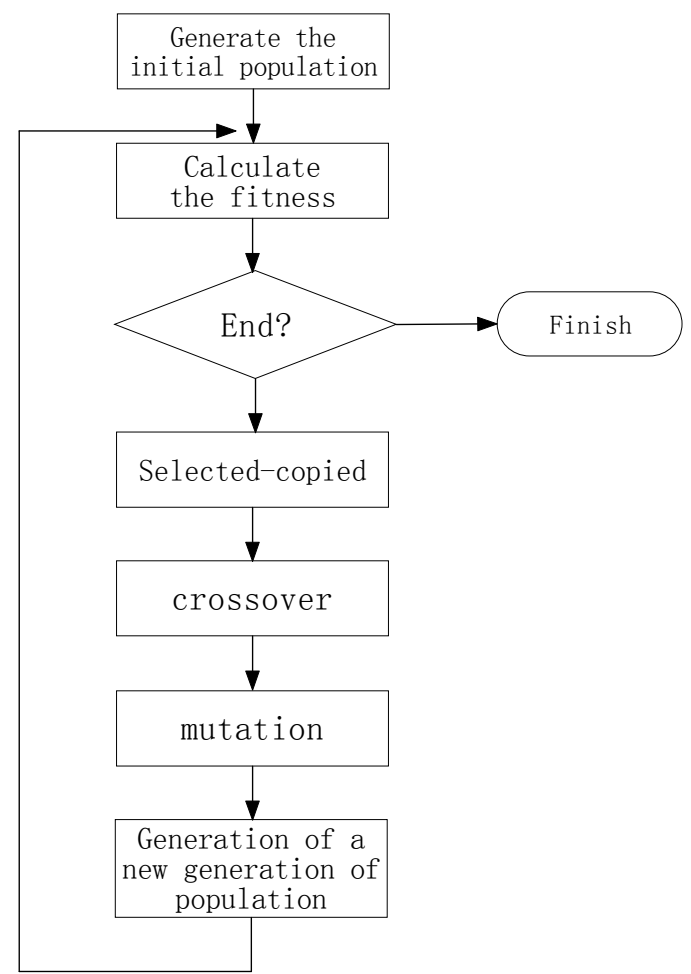

FIGURE III. THE BASIC FLOW FRAME OF GENETIC ALGORITHM

The improved GA [12] divides the initial population into multiple subpopulations, and assigns a sub-target to each subpopulation by individual selection techniques such as parallel selection. The integration of a single optimal solution to meet the overall goal constraints. However, the parallel selection method is the average allocation of the sub-target, and can't meet the requirements of solving the problem according to a certain order and priority scheduling resources. In this paper, the multi-objective constraints of resource scheduling is analyzed in detail, and the parallel selection method is improved. The multi-objective selection method of priority guidance is used to solve the optimal solution. The improved genetic algorithm used in this study is shown in Figure 4.

In addition to using a good algorithm, the algorithm uses a variety of genetic operations such as cross thinking to explore good computing methods and mutation of sudden change. It is a multi-group GA of high analysis ability.

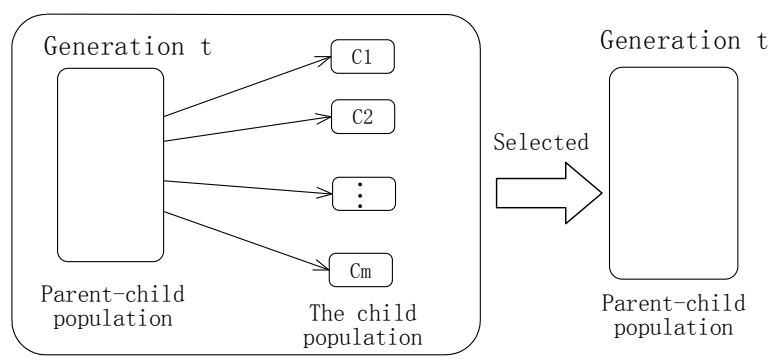

FIGURE IV. IMPROVED GENETIC ALGORITHM

\section{DERIVATION OF THE CALCULATION FORMULA OF THE SIZE VALUE}

First of all, the control point coordinates of 1-7 are used as the object of the size calculation formula, and it is implemented five times with a genetic algorithm. In the process of implementation, the best size calculation formula used for those who were measured, and calculate.

Here, the number of suitable control points need be determined. If the number of control points used by the size calculation formula is increased, a better analysis result can be obtained. However, it is not always possible to obtain a better analysis result if the number of control points used in the size value estimation formula is increased, when the obtained size calculation formula calculate the size of the non-measured person. There is the reason: if the number of control points is too large, the size calculation formula of the noise containing the measured noise is derived, which has a negative effect on the estimated result. The appropriate number of control points can be estimated for ordinary subjects, when a size value is used as the object size calculation formula.

In this study, the size calculation formula for the other four items is derived using the same method like height. In the following $(\mathrm{I}) \sim(\mathrm{V})$, the obtained size calculation formula uses for the unknown 25 subjects. And the relationship between the measured value and the calculation value is shown in Table 1.

(I)The calculation formula of the height size

$$
\mathrm{D}=129.667-0.031 \cdot \mathrm{X}_{271}+0.109 \cdot \mathrm{Y}_{127}
$$

(II) The calculation formula of the waistline size

$\mathrm{D}=248.464+0.039 \cdot \mathrm{X}_{1}-0.081 \cdot \mathrm{X}_{127}+0.162 \cdot \mathrm{X}_{389}-0.134 \cdot \mathrm{X}_{298}{ }^{+}$ $0.171 \cdot Z_{387}-0.126 \cdot Z_{337}$

(III)The calculation formula of the weight size

$\mathrm{D}=-579.709-0.127 \cdot \mathrm{X}_{297}+0.022 \cdot \mathrm{Y}_{30}+0.234 \cdot \mathrm{Z}_{142}-0.358 \cdot \mathrm{Z}_{147}$

(IV)The calculation formula of the surface area size

$$
\begin{gathered}
\mathrm{D}=-57.902-0.011 \cdot \mathrm{X}_{291}+0.012 \cdot \mathrm{X}_{199}+0.004 \cdot \mathrm{Y}_{63}{ }^{-} \\
0.013 \cdot \mathrm{Y}_{431}+0.015 \cdot \mathrm{Z}_{348}-0.118 \cdot \mathrm{Z}_{467}+0.033 \cdot \mathrm{Z}_{394}
\end{gathered}
$$

(V) The calculation formula of the volume size

$\mathrm{D}=-41.211-0.010 \cdot \mathrm{X}_{517}+0.003 \cdot \mathrm{Y}_{129}-0.012 \cdot \mathrm{Z}_{409}+0.032 \cdot \mathrm{Z}_{409}$ 
TABLE I. ANALYSIS OF THE CALCULATION FORMULA OF THE SIZE VALUE

\begin{tabular}{|c|c|c|c|c|c|c|c|c|}
\hline \multirow{3}{*}{$\begin{array}{l}\begin{array}{c}\text { The number of } \\
\text { the formula of } \\
\text { the size }\end{array} \\
\text { (I) }\end{array}$} & \multirow{3}{*}{$\begin{array}{c}\begin{array}{c}\text { The name } \\
\text { of the size }\end{array} \\
\text { height }\end{array}$} & \multirow{3}{*}{$\begin{array}{c}\begin{array}{c}\text { The number } \\
\text { of control } \\
\text { points }\end{array} \\
3 \\
\end{array}$} & \multicolumn{3}{|c|}{ Analysis $(\mathrm{N}=60)$} & \multicolumn{3}{|c|}{ Calculation $(\mathrm{N}=25)$} \\
\hline & & & $r$ & $Q \quad m$ & & $r$ & $\max$ & \\
\hline & & & 0.985761 & 7.66 & 17.00 & 0.998508 & 4.70 & 8.66 \\
\hline (II) & waistline & 7 & 0.989151 & 12.43 & 32.80 & 0.994334 & 12.372 & 22.47 \\
\hline (III) & weight & 5 & 0.977751 & 17.19 & 44.90 & 0.954486 & 23.52 & 31.63 \\
\hline (IV) & surface area & 8 & 0.994721 & 0.54 & 1.37 & 0.966287 & 1.12 & 2.52 \\
\hline$(\mathrm{V})$ & volume & 3 & 0.980689 & 1.08 & 3.31 & 0.982435 & 1.22 & 2.52 \\
\hline
\end{tabular}

In the Table, the characters refer to as the following meaning, that is, "r": The correlation coefficient between the calculation value (regression value) and the measured value. "Q": the error of each subject *. "maxQ": in the group of subjects, the value of the subject is that the difference between the calculated value and the measured value is the largest. Error unit: (III) weight $(0.1 \mathrm{~kg})$, (IV) surface area $\left(100 \mathrm{~cm}^{2}\right)$, (V) volume $\left(1000 \mathrm{~cm}^{3}\right)$, and others $(\mathrm{mm}) . \mathrm{N}$ : analysis of 60 people, calculations of 25 people.

According to the results, if the calculation error of the height is $4.70(\mathrm{~mm})$, the calculation error of the waistline is $12.37(\mathrm{~mm})$, the calculation error of the surface area is $1.12(100 \mathrm{~cm} 2)$, and the calculation error of the volume is $1.22(1000 \mathrm{~cm} 3)$, the size value of unknown subjects can be calculated accurately. However, the calculation error of the weight is relatively large, namely: $\mathrm{Q}=2.35(\mathrm{~kg})$, maxQ=3.16 $(\mathrm{kg})$. The weight should include the whole body weight, and the body model is only the main body, so it is difficult to express accurately. Generally, the body volume and weight can use the same value, because the volume can be accurately calculated.

\section{CONCLUSIONS}

In this paper, the B-Spline surface shape model was created for human body size estimation by linear combining many data such as body height, weight, chest size, the body surface area and volume. Then several control points were selected by GA to select best points in theses dataset, and mathematical model of estimating human body size was built. Experimental results indicated that this model has advantages of high efficiency and low error rates in estimating human body size.

According to the results, the size values of two-dimensional and three-dimensional size, such as body surface area and volume, can be represented by linear combination of the control points coordinates. After that, the same method is used to measure the size of the unmeasured value. It is a subject to be studied about the relevant measurement in the future. For example, the measurement of human body density can be analyzed according to the weight of the shore and the body weight of water. This method is called in-water weight measurement method. If this method is used to consider the volume and weight, the body density will be obtained. At the same time, it is estimated that the body fat rate.

\section{ACKNOWLEDGMENT}

The authors acknowledge the Special Training Plan Project of Xinjiang Uyghur Autonomous Region's Minority Science and Technological Talents (No. 201323121).

\section{REFERENCES}

[1] Y. Zheng, H. Liu, J. Dorsey and N. J. Mitra, Ergonomics-Inspired Reshaping and Exploration of Collections of Models, IEEE Transactions on Visualization and Computer Graphics. 6 (2016) 1732-1744.

[2] ZHANG Chun-hong, LIN Da-quan, Parameters research on the adult anthropomorphic phantom of china, Machinery. 7 (2009) 1-3.

[3] JONES P R M, WEST G M, The lough borough anthropometric shadow scanner (LASS), Endeavor. (13) 1989 162-168.

[4] Li Jian, Xu Xueli, Lan Xueyuan, Zhang Chengyan, Feature Extraction and Measurement of the Human Body Based on Harris Corner Detection, Computer Measurement \& Control. 2 (2014) 367-369.

[5] LI Fu-xing, SUN Jian, Research on correction and reconstruction methods of human dimensions in human-machine engineering, Journal of Machine Design. 4 (2015) 116-120.

[6] HAN Li, LI Lin, XU Jian-Guo, TANG Di, Structure Analysis for Human Models Based on Surface and Spatial Features, PR \& AI. 3 (2015) 231238.

[7] Li Jun, Zhang Mingmin , Pan Zhigeng , Wang Shengbo, Yan Zheng, Creating real body model of dressed human based on fat extent of body, Multimed Tools Appl. 74 (2015) 6951-6966.

[8] S. Zeitvogel, A. Laubenheimer, Fitting a deformable 3D human body model to depth images using convolutional neural networks, 2016 12th IEEE International Symposium on Electronics and Telecommunications (ISETC), Timisoara, 2016, pp. 321-326.

[9] Ayman A. Nada, Use of B-spline surface to model large-deformation continuum plates: procedure and applications, Nonlinear Dyn. 72 (2013) 243-263.

[10] Ahmed A. EL- Sawy, Mohamed A. Hussein1, EL-Sayed M. Zaki, A. A. Mousa, An Introduction to Genetic Algorithms: A survey A practical Issues, International Journal of Scientific \& Engineering Research. 1 (2014) 252-262.

[11] Garcia-Capulin, C.H., Cuevas, F.J., Trejo-Caballero, G.,Rostro-Gonzalez, $\mathrm{H}$, Hierarchical Genetic Algorithm for B-Spline Surface Approximation of Smooth Explicit Data, Mathematical Problems in Engineering, 1 (2014) 1-11.

[12] Marco Altini, Julien Penders and Oliver Amft, Body Weight-Normalized Energy Expenditure Estimation Using Combined Activity and Allometric Scaling Clustering, 35th Annual International Conference of the IEEE EMBS, Osaka, Japan, 2013, pp. 3 - 7. 